\title{
Value of serum and follicular fluid sirtuin (SIRT)1 and SIRT2 protein levels in predicting the outcome of assisted reproduction
}

\author{
Ling-Nv Yao ${ }^{1 \#}$, Tian-Fang Zhang ${ }^{2 \#}$, Wen-Qin Lin ${ }^{1}$, Nan Jiang ${ }^{1}$, Hai-Feng Cao ${ }^{1}$, Hong Li $^{3}$, \\ Jian-Hua Qian ${ }^{4 \wedge}$ \\ ${ }^{1}$ Reproductive Medical Center, First Affiliated Hospital, School of Medicine, Zhejiang University, Hangzhou, China; ${ }^{2}$ Department of Rehabilitation \\ Medicine, The First Affiliated Hospital, School of Medicine, Zhejiang University, Hangzhou, China; ${ }^{3}$ Surgical Department, First Affiliated Hospital, \\ School of Medicine, Zhejiang University, Hangzhou, China; ${ }^{4}$ Department of Gynaecology, First Affiliated Hospital, School of Medicine, Zhejiang \\ University, Hangzhou, China \\ Contributions: (I) Conception and design: LN Yao; (II) Administrative support: JH Qian; (III) Provision of study materials or patients: WQ Lin; (IV) \\ Collection and assembly of data: N Jiang, HF Cao, H Li; (V) Data analysis and interpretation: TF Zhang; (VI) Manuscript writing: All authors; (VII) \\ Final approval of manuscript: All authors. \\ \#These authors contributed equally to this work. \\ Correspondence to: Prof. Jian-Hua Qian. Department of Gynaecology, First Affiliated Hospital, School of Medicine, Zhejiang University, Hangzhou \\ 310003, China. Email: qianjianhua@zju.edu.cn.
}

Background: To explore whether serum and follicular fluid (FF), sirtuin 1 (SIRT1), and SIRT2 could predict the outcome of assisted reproduction.

Methods: All patients underwent in vitro fertilization (IVF)/intracytoplasmic sperm injection (ICSI) for the first time in the Reproductive Medicine Center of the First Affiliated Hospital of Zhejiang University Medical College from March 2018 to December 2018. According to cumulative clinical pregnancy outcomes, the patients were divided into a pregnancy group and non-pregnancy group. We measured the serum levels of SIRT1, SIRT2, anti-Müllerian hormone (AMH), follicle-stimulating hormone (FSH), luteinizing hormone (LH), and estradiol (E2) from the second to the fifth day of menstruation, and the levels of SIRT1 and SIRT2 in serum and FF on the day of human chorionic gonadotropin (HCG) injection and oocyte retrieval.

Results: A total of 125 patients met the inclusion criteria. The pregnancy group comprised 56 cases and non-pregnancy group 69 cases. There were significant differences in basal level SIRT2 (bSIRT2), AMH, antral follicle count (AFC), number of oocytes obtained, number of mature eggs, number of fertilized eggs, number of excellent embryos, number of blastocyst formations, and number of transferred high-quality embryos between the two groups. The area under the curve (AUC) values of bSIRT2, AFC, AMH, and age were significantly different from those under the opportunity reference line $(\mathrm{P}<0.05)$. In the subsequent correlation analysis, FFSIRT2, and HCG day serum SIRT2 were negatively correlated with age $(\mathrm{r}=-0.35$, $\mathrm{r}=-0.19)$, and positively correlated with AFC ( $\mathrm{r}=0.2, \mathrm{r}=0.02)$. Serum SIRT1 on HCG day was negatively correlated with the number of blastocysts and the number of frozen embryos $(r=-0.18, r=-0.21)$. Levels of FF SIRT1 and FF SIRT2 were significantly lower than those in serum SIRT1 and SIRT2, and there was no significant difference in serum SIRT1 and SIRT2 before and after ovulation promotion.

Conclusions: The results suggest that bSIRT2 has significant statistical significance in predicting the cumulative number of pregnancies. When combined with AMH, AFC, and age, bSIRT2 can predict the cumulative pregnancy outcome. In addition, the level of serum SIRT1 and SIRT2 were not affected by ovulation promotion.

Keywords: SIRT1; SIRT2; anti-Müllerian hormone (AMH); fertility outcomes; assisted reproduction

\footnotetext{
^ ORCID: 0000-0003-1320-6860.
} 
Submitted Dec 10, 2020. Accepted for publication Jan 29, 2021.

doi: 10.21037/atm-21-63

View this article at: http://dx.doi.org/10.21037/atm-21-63

\section{Introduction}

As environmental factors, social pressure, an increase in the childbearing age of women, and decreasing fertility rates intertwine, the demand for assisted reproductive technology is increasing. However, the cost of assisted reproductive technology is high, and the cycle is long and unpredictable. Methods to evaluate the reproductive outcome of assisted reproductive technology are of great significance for clinical guidance. Patients of advanced age and decreased ovarian reserve (DOR) are more likely to be infertile and are often predicted to have poor reproductive outcomes. Generally, with increasing age, the number of mitochondria in the cytoplasm of oocytes gradually decreases, ATP content decreases, and DNA variation and aneuploidy increase, while the number of follicles and oocytes, and embryo quality decreases. However, because of differences in the size of individual follicle pools, the speed of follicular atresia, heredity factors, environment impacts, a history of ovarian surgery, and other factors, ovarian reproductive age is often inconsistent with actual age. At present, serum antiMüllerian hormone (AMH) and antral follicle count (AFC) are considered as the earliest and most accurate diagnostic indices of premature ovarian failure before the symptoms of ovarian reserve decline $(1,2)$. However, the value of $\mathrm{AMH}$ and $\mathrm{AFC}$ in predicting oocyte and embryo quality is uncertain (3). There are still no accepted indicators for predicting oocyte quality, embryo development potential, and assisted reproductive outcomes.

Members of the sirtuin (SIRT) family are deacetylases. Sirtuins play different roles in regulating metabolism, cell proliferation, and genomic stability and are related to aging and age-related diseases in mammals, including cancer, metabolic disorders, and neurodegenerative diseases. Seven members of this family have been identified so far and named SIRT1-7 (4).

Mammalian SIRTs can interact with metabolic responses to regulate to nutritional input in multiple tissues and organs, and then affect energy metabolism and change the function of cancer stem cells. SIRT1 inhibits glycolysis through direct suppression of the hypoxia-inducible factor $1 \alpha(\mathrm{HIF} 1 \alpha)$ with deacetylation (5). Meanwhile, during the early fasting state, SIRT1 suppresses gluconeogenesis via
CREB-regulated transcription coactivator 2 (CRTC2) deacetylation, resulting in CRTC2 deprivation and later decrement in transcription of gluconeogenic genes (6). SIRT1 also regulates lipid metabolism through the modification of liver X receptor (LXR), farnesoid X receptor (FXR), and sterol regulatory element binding protein (SREBP) (7). SIRT2 can be involved in the regulation of biological functions including glucose and lipid metabolism through deacetylation of different endogenous substrates. SIRT2 regulates insulin mainly through phosphatidylinositol 3 kinase (PI3K)/protein kinase B (KB/Akt) signaling pathway (8). Recent studies (9) have shown that SIRT2 can enhance the activity of three enzymes by deacetylation, namely pyruvate kinase (PK), phosphoenolpyruvate carboxylase (PEPCK), and phosphoglycerate mutase (PGAM), thus promoting the tricarboxylic acid cycle.

There are also many studies suggesting that SIRT1 and SIRT2 have many correlations with nervous system diseases. SIRT1 has a protective effect in the nervous system. Through deacetylation, it can be used to target proteins, such as p53, forkhead transcription factor o, peroxisome proliferator activated receptor gamma coactivator $1 \alpha$, and protein kinase $\mathrm{B}$, which can affect apoptosis factors, neuroinflammation, oxidative stress, and mitogenesis, so as to achieve the regulation of neuronal apoptosis (10-12). Recent research indicates that is expected to become a new target for the treatment and prevention of neurodegenerative diseases. For instance, SIRT2 was shown to play a partial role in lipopolysaccharide-induced neuroinflammation (13); SIRT2 inhibitor AK7 was found to have neuroprotective effects in a Parkinson disease model (14); SIRT2 in the hippocampus may participate in the regulation of antidepressant like behavior by regulating neurogenesis; SIRT2 may regulate adipocyte differentiation by regulating FoxO1 acetylation/deacetylation activity $(15,16)$. Other studies have shown that SIRT2 can regulate RIP1/RIP3 signaling pathway in programmed necrosis (17). As can be seen, SIRT2 is an important regulator of programmed necrosis and a promising drug target in the prevention of necrotic injury.

In 2003, a study found that male and female mice in which SIRT1 was knocked out had reproductive phenotype 
defects (18). Since then, SIRT's role in regulating mammalian reproductive function has gradually attracted attention. SIRT1 is involved in the processes of DNA repair, aging and apoptosis. SIRT2 and SIRT6 are involved in the assembly of microtubules and the process of oocyte division. SIRT3 is the most studied mitochondrial protein; it can affect key mitochondrial functions, regulate cell generation and removal of reactive oxygen species (ROS) and the production of adenosine triphosphate (ATP), and exert antioxidant effects. However, SIRT4, SIRT5, and SIRT7 have been given relatively little attention in the field of reproduction.

Reproductive defects caused by ovarian aging, oxidative stress in oocytes, and high-calorie diets are challenging the fertility of modern women. During in vitro fertilization and embryo transfer (IVF-ET) treatment, repeated ovulation induction, the oxidative stress of oocytes in vitro, and the damage to the quality of oocytes caused by an excitable state cannot be ignored. Preclinical and clinical studies have provided significant evidence that strategies aimed to improve SIRT expression or activity counteract deleterious effects on fertility of polycystic ovary syndrome (PCOS), endometriosis, diabetes, xenobiotic stress, and aging (19). Studies by Selenay and others have shown that metformin combined with resveratrol can induce antioxidant and anti-inflammatory effects by activating SIRT1 and AMPK [adenosine 5'-monophosphate (AMP)-activated protein kinase], so as to improve weight gain, and abnormal endocrine and polycystic ovarian changes in PCOS patients $(20,21)$. SIRT1 also has been suggested to be a key driver of the progesterone resistance contributing to pathophysiology and survival of ectopic lesions and has been proposed as a valuable endometrial marker in women with endometriosis (22).

Studies have shown that mild oxidative stress can upregulate the expression of SIRT1, thus inducing the response of cells to the change of redox state, while severe oxidative stress can increase the degradation of SIRT1, thus inducing apoptosis (23). The targets of SIRT1 in response to oxidative stress include tumor suppressor gene p53, transcription factor FOXOS, and nuclear factor NF-kAPAB (NF-kappa B), which play different roles in mediating SIRT1 response to the redox state $(24,25)$.

SIRT1 is also considered to be the key protein responsible for delaying the aging process in animal models. Upregulation of SIRT1 protein showed a better resistance to age-related phenotypes, while downregulation of SIRT1 accelerated aging (26). Some gene modified mouse experiments have proven that some genes related to ovarian reserve dynamics interact with SIRT signals, such as FOXO3a, as the action site of SIRT1 and SIRT3, and its inhibitory effect can accelerate the depletion of preantral follicles; mTOR signal transduction pathway is affected by SIRT1 and SIRT2, and its inhibitory effect can cause follicle dormancy (27-29). Ovarian aging is characterized by a gradual decrease in the number of follicles and oocyte quality. Zhang (30) found that the expression levels of SIRT1, SIRT3, and SIRT6 were significantly decreased in the ovaries of aged mice and mice receiving chemotherapy, and were positively correlated with the number of primary follicles. These results suggest that SIRT1, SIRT3, and SIRT6 are closely related to ovarian reserve.

SIRT2 participates in the mediation of mitosis, oxidative stress, microtubule movement, chromatin agglutination, and cell movement (31). Zhang et al. (32) suggested that the level of SIRT2 protein in the oocytes of aged mice is significantly decreased, and the age-related meiotic defect could be improved after overexpression of SIRT2; furthermore, SIRT2 may be involved in the formation or assembly of spindle. Subsequently, Qiu et al. (33) confirmed that the specific deletion of SIRT2 could lead to damage of the kinetochore-microtubule junction of mouse oocytes, thus destroying the maturation process of oocytes and the spindle/chromosome tissue.

In addition, SIRTs are highly expressed in mammalian testicular tissue (34). In particular, SIRT1 has been detected in the nuclei of spermatogonia, spermatocytes, and round spermatids, suggesting an intrinsic direct activity of SIRT1 in developing male germ cells during spermatogenesis (18). Mutant mice (SIRT1 knockout mice) were found to display infertility with decreased testes size, but the rare spermatozoa that recovered from their cauda epididymis were immotile, exhibited abnormal morphology, and were not as efficient in IVF experiments as wild-type sperm, due to a spermatogenesis arrest in late-meiotic prophase with degenerating or dying spermatocytes. This was associated with severe morphological abnormalities, apoptotic features, and increased DNA damage within the seminiferous epithelium (35).

SIRTs participate in the regulation of mammalian reproductive functions through multidirectional targets. The clinical application of SIRTs agonists may delay the reproductive life of elderly women or patients with low ovarian reserve, reduce the aging of oocytes after ovulation, combat the oxidative stress faced by oocytes during the IVF cycle, promote sperm production, etc. Consequently, 
SIRTs may have great therapeutic potential in the field of reproductive medicine in the future.

At present, few studies exist concerning the relationship between SIRT protein levels in serum and follicular fluid (FF) and reproductive outcomes. A study by Bódis (36) suggested that serum SIRT1 and SIRT6 may play a role in oocyte maturation and clinical pregnancy IVF embryo transfer, and are related to human reproduction. However, this study included only a small number of patients and did not assess the value of SIRT2. The purpose of the present study was thus to investigate the predictive value of serum and FF SIRT1 and SIRT2 protein levels on assisted reproductive outcomes.

We present the following article in accordance with the STARD reporting checklist (available at http://dx.doi. org/10.21037/atm-21-63).

\section{Methods}

\section{Cases}

The study population comprised 125 patients who underwent their first cycles of IVF/intracytoplasmic sperm injection (ICSI) treatment from March 2018 to December 2018 at the Reproductive Medicine Center of the First Affiliated Hospital of Zhejiang University School of Medicine. This study was approved by the Research Ethics Committee of the First Affiliated Hospital, College of Medicine, Zhejiang University (No. 2017-734) and written consent was obtained from all the patients. All procedures performed in this study involving human participants were in accordance with the Declaration of Helsinki (as revised in 2013).

The exclusion criteria were as follows: (I) PCOS patients; (II) patients with endometriosis stage II or above or adenomyosis; (III) patients with endocrine diseases such as hyperprolactinemia or thyroid diseases; (IV) patients with immune system diseases; (V) patient history of repeated implantation failure; (VI) patients with uterine cavity adhesion, endometrial polyps, or other factors affecting the history of embryo implantation; (VII) patient surgical history of genital malignant tumors; (VIII) patient with uterine malformation; (IX) and patients with contraindications to IVF.

\section{Hormone measurements}

The concentration of basal serum SIRT1 and SIRT2
(bSIRT1 and bSIRT2), AMH, follicle-stimulating hormone (FSH), luteinizing hormone (LH), and estradiol (E2) were measured on the second to fifth day of menstruation. The concentration of serum SIRT1 and SIRT2 were measured again on the day of human chorionic gonadotropin (HCG) injection, while FF SIRT1 and SIRT2 were determined on the day of oocyte pick up (OPU). Serum AMH, SIRT1, and SIRT2, and FF SIRT1 and SIRT2 were measured by enzyme-linked immunosorbent assay (ELISA). The ELISA test kits were obtained from the Shanghai Yuduo Company (China).

\section{Clinical and laboratory data collection}

The number of antral follicles with a diameter of $2-$ $8 \mathrm{~mm}$ in bilateral ovaries was recorded by transvaginal ultrasonography in our center between the second to fifth days of menstruation. Other data collected included infertility years, age, factors of infertility, body mass index (BMI), number of eggs, fertilization rate, rate of highquality embryos, blastocyst formation rate, cumulative clinical pregnancy rate, and live birth rate.

\section{Ovulation stimulation program}

An ovulation promotion program was selected according to the individual ovarian function of patients and included long-term luteal regimen, short-term regimen, microstimulation, and natural cycle regimen. Vaginal ultrasound monitored follicular development. If there were 3 or more follicles 16 to $18 \mathrm{~mm}$ in diameter and a consistent rise in serum estradiol concentration, $\mathrm{Gn}$ was discontinued. One HCG 4,000-10,000 IU or rhHCG (250 $\mu \mathrm{g} / \mathrm{branch})$ injection was given at night, and OPU was performed 34 36 hours after injection.

\section{Definition of relevant indicators}

High-quality embryos, clinical pregnancy, and cumulative pregnancy rate were considered the relevant indicators. High-quality embryos were consider those having blastomeres basically uniform in size, regular in shape, homogeneous in cytoplasm or granular cells, with debris less than $20 \%$. The criteria for clinical pregnancy were the following: in addition to biochemical indicators, there was clinically visible evidence of pregnancy, including an intrauterine pregnancy sac, ectopic pregnancy, abortion, or visible curettage on chorionic tissue. Finally, the cumulative 
pregnancy rate was considered to be the percentage of successful pregnancies in the total number of egg retrieval cycles after all embryos had been in transplanted in an egg retrieval cycle.

\section{Statistical analysis}

SPSS 19.0 statistical software (IBM Corp., USA) was used for data analysis. Quantitative data are expressed as a mean \pm standard deviation, and qualitative data are expressed as counts or percentages. Comparison of quantitative variables between two groups were performed using 2-tailed Student's $t$-test, and comparison of qualitative variables was performed using the chi-square test. To correlate the level of SIRT1 and SIRT2 with other parameters such as age, $\mathrm{AMH}, \mathrm{AFC}$, and the number of eggs, data were analyzed with the Pearson correlation coefficient. Serum and FF SIRT1 and SIRT2, serum AMH, FSH, AFC, and age were used to construct the receiver operator characteristic curve (ROC curve) to evaluate the value in predicting cumulative fertility outcomes. A $\mathrm{P}$ value $<0.05$ was considered statistically significant. This was a prospective study with a small sample size, therefore small sample size calculations (PASS11, NCSS, USA) were undertaken to determine the power of the study to detect differences at the 0.05 probability level. Values of $\mathrm{P}<0.05$ were considered statistically significant.

\section{Results}

\section{Analysis of the differences of laboratory and clinical related indicators between pregnancy and non-pregnancy groups}

According to the cumulative pregnancy outcome, patients were divided into a pregnancy group (56 cases) and a nonpregnancy group (69 cases). The levels of bSIRT2, AMH, age, and AFC, along with the egg number, fertilization number, number of high-quality embryos, blastocyst culture number, blastocyst formation number, and number of transplanted high-quality embryos in the pregnant group were significantly higher than those in the non-pregnant group (Table 1).

\section{ROC curve analysis of serum and FF SIRT1 and SIRT2 protein levels to predict the outcome of assisted reproduction pregnancy}

Figures 1 and 2 show the ability of FF SIRT1 and FF SIRT2 levels, HCG day serum SIRT1 and SIRT2, bSIRT1 and bSIRT2, AFC, AMH, age, and basic FSH to predict the cumulative pregnancy rate. The respective areas under the ROC curve were as follows: $0.478,0.529,0.522,0.594$, $0.543,0.640,0.667,0.686,0.632$, and 0.514 , respectively. The results showed that the area under the curve AUC values of bSIRT2, AFC, AMH, and age were significantly different from the area under the opportunity reference line $(\mathrm{P}<0.05)$ (Table 2).

Figure 3 indicates that FF SIRT1 and FF SIRT2, HCG daily serum SIRT1 and SIRT2, bSIRT1 and bSIRT2, $\mathrm{AFC}, \mathrm{AMH}$, age, and basic FSH had no statistical value in predicting clinical pregnancy and live birth in assisted reproduction.

\section{Logistic regression analysis of factors related to cumulative pregnancy}

There are many factors related to infertility. To reduce deviation, this study strictly screened the patient's etiology before enrolment and excluded those with endocrine abnormalities such as hyperandrogenemia, hyperprolactinemia, PCOS, thyroid dysfunction, and endometrial abnormalities, as well as ectopic disease and immune abnormalities. To further clarify the interfering factors affecting cumulative pregnancy, logistic regression analysis was used to perform regression analysis on related indicators. It was found that only bSIRT2 was statistically significant $(\mathrm{P}=0.049)$, while $\mathrm{AMH}, \mathrm{AFC}$, age, and $\mathrm{BMI}$ were not statistically significant (Table 3).

\section{Correlation analysis between serum and FF SIRT1 and FF SIRT2 protein levels and some laboratory and clinical related indicators}

Table 4 shows that FF SIRT 2 and FF SIRT 2 (HCG day) were negatively correlated with age $(\mathrm{r}=-0.35, \mathrm{r}=-0.19)$ and FF SIRT2 was positively correlated with $\mathrm{AFC}(\mathrm{r}=0.2)$. Serum SIRT1 on the day of HCG injection was negatively correlated with the number of blastocyst formations and frozen embryos $(\mathrm{r}=-0.18, \mathrm{r}=-0.21)$; meanwhile, serum SIRT2 on the day of HCG injection was positively correlated with AFC, the number of eggs obtained, mature eggs, fertilizations, blastocyst formations, and frozen embryos $(r=0.18, r=0.22, r=0.22, r=0.22, r=0.21, r=0.20$, respectively), and was negatively correlated with basic E2 $(\mathrm{r}=-0.18)$. 
Table 1 Comparison of clinical and laboratory indices between cumulative pregnant and non-pregnancy groups

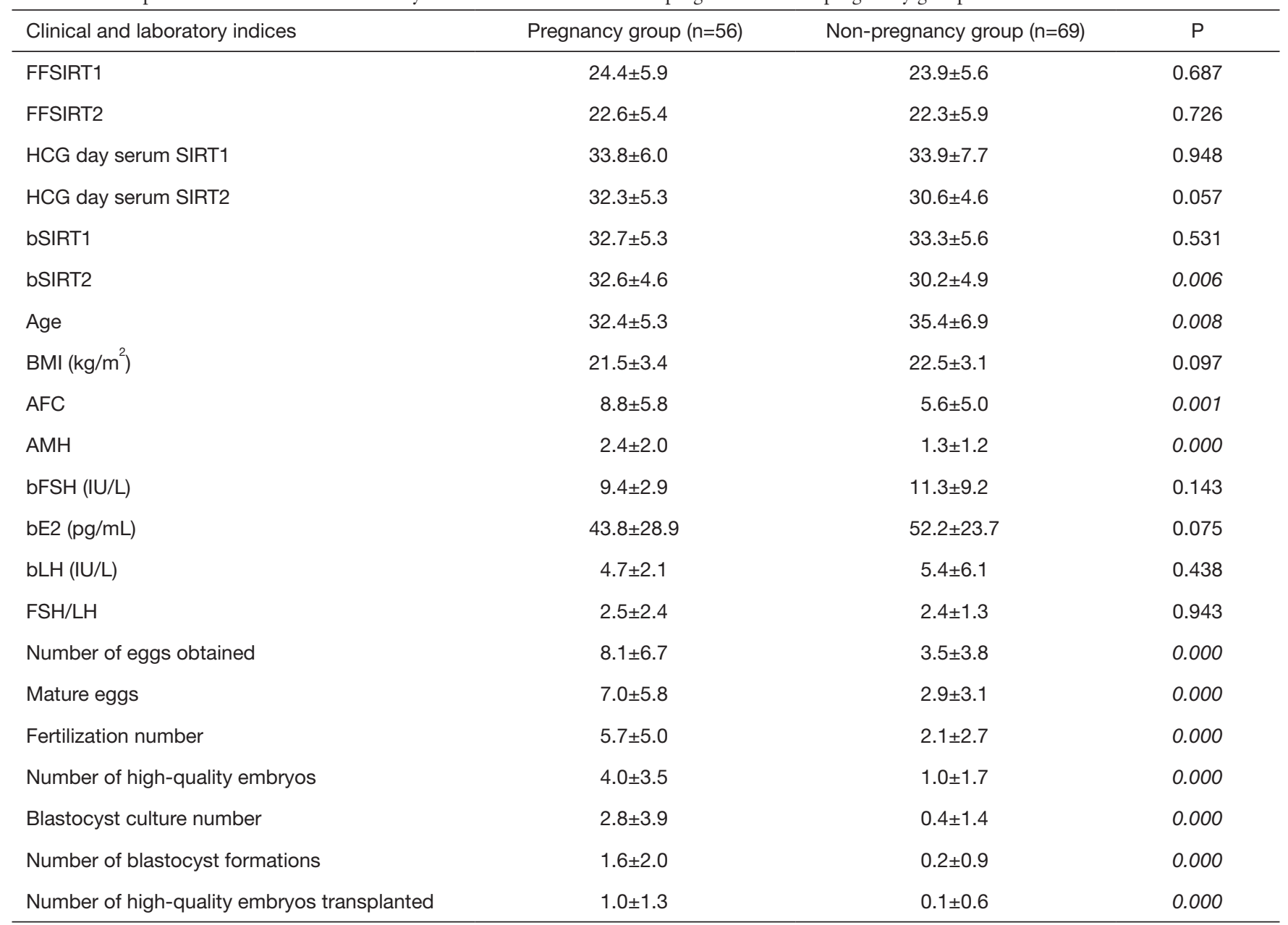

Statistical significance was evaluated using Student's $t$-test. Statistically significant differences $(P<0.05)$ are marked in italics type. $\mathrm{AMH}$, anti-Müllerian hormone; AFC, antral follicular count; BMI, body mass index; bFSH, base level of follicle-stimulating hormone; LH, Luteinizing hormone; FFSIRT1, follicular fluid SIRT1; FFSIRT2, follicular fluid SIRT2; HCG day SIRT1, HCG injection day serum SIRT1; HCG day Sirt2, HCG injection day serum SIRT2; bSIRT1, base level of serum SIRT1; bSIRT2, base level of serum SIRT2; SIRT, sirtuin; HCG, human chorionic gonadotropin.

\section{Changes in serum and FF SIRT1 and FF SIRT2 levels and their correlation during assisted reproduction}

Table 5 indicates that the bSIRT1, bSIRT2, and HCG day serum SIRT1 and SIRT2 had no significant differences, while the FF SIRT1 and FF SIRT2 levels were significantly lower than the serum SIRT1 and SIRT2 levels. There was no significant correlation among bSIRT1, bSIRT2, HCG day serum SIRT1 and SIRT2, and FF SIRT1 and FF SIRT2.

\section{Discussion}

Currently, most of the indicators predicting fertility outcomes, such as $\mathrm{AMH}, \mathrm{AFC}$, and age, are those predicting ovarian reserve and response. However, their value is uncertain. SIRT1 and SIRT2 proteins are important members of the SIRT protein family of deacetylases, and the role of energy receptors in various fields, such as cell biology, metabolism, and cell fate, is critical. Previous studies have shown that under calorie restriction conditions, an increase in endogenous SIRT1 reduces the expression of $\mathrm{p} 53$ (instead of FOXO3a), reduces follicular apoptosis or atresia, and maintains ovarian reserve (37). SIRT2 is involved in the mitotic structure, from the central body of the premitotic stage to the central body 
ROC curve

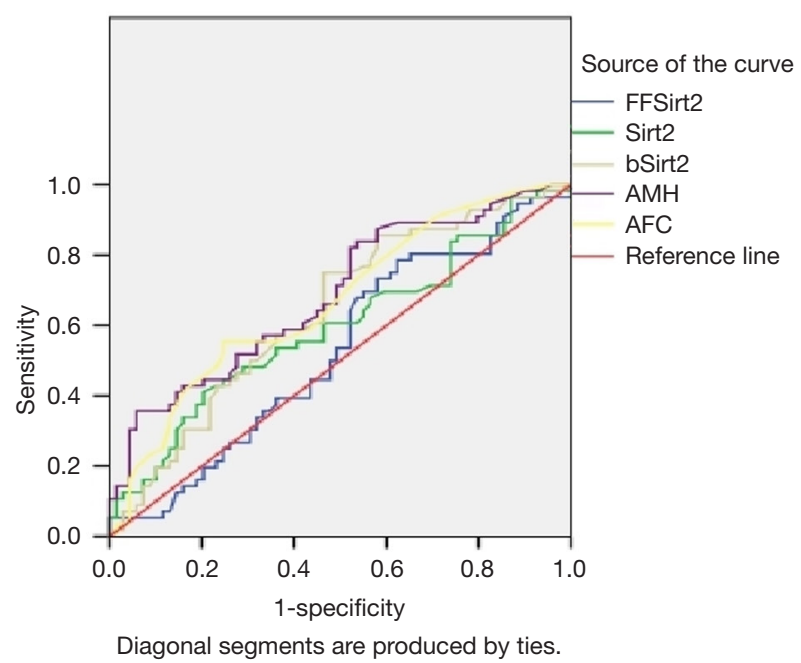

CR-AUC

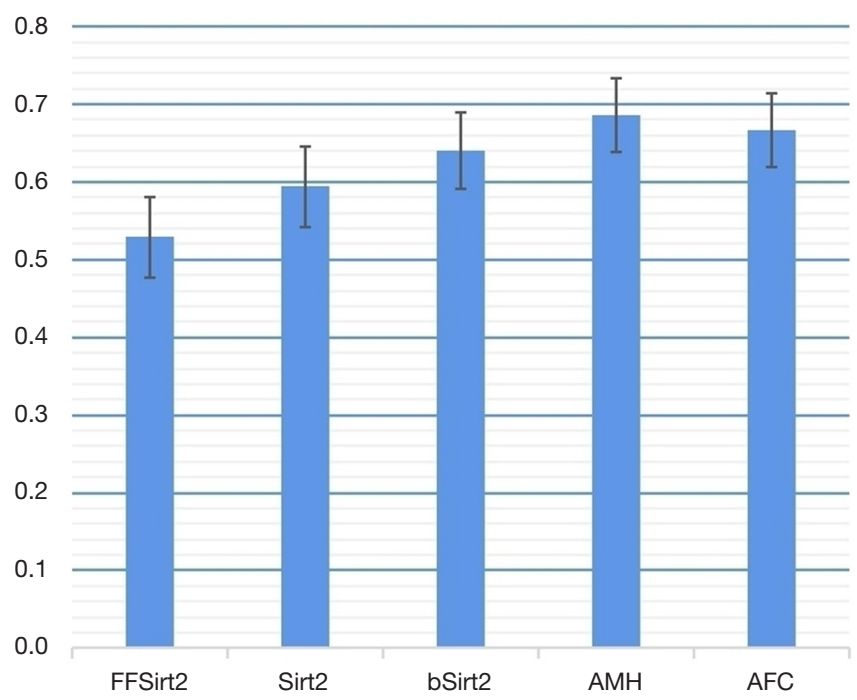

Figure 1 The area under the curve values of bSIRT2, AFC, and AMH in predicting cumulative clinical pregnancy were significantly different from the area under the opportunity reference line $(\mathrm{P}<0.05)$. AFC, antral follicular count; AMH, anti-Müllerian hormone; bSIRT2, base level of serum SIRT2; CR, cumulative pregnant rate hormone; FFSIRT2, follicular fluid SIRT2; SIRT, sirtuin; SIRT2, HCG injection day serum SIRT2; AUC, area under ROC curve; ROC, receiver operating characteristic curve.

ROC curve

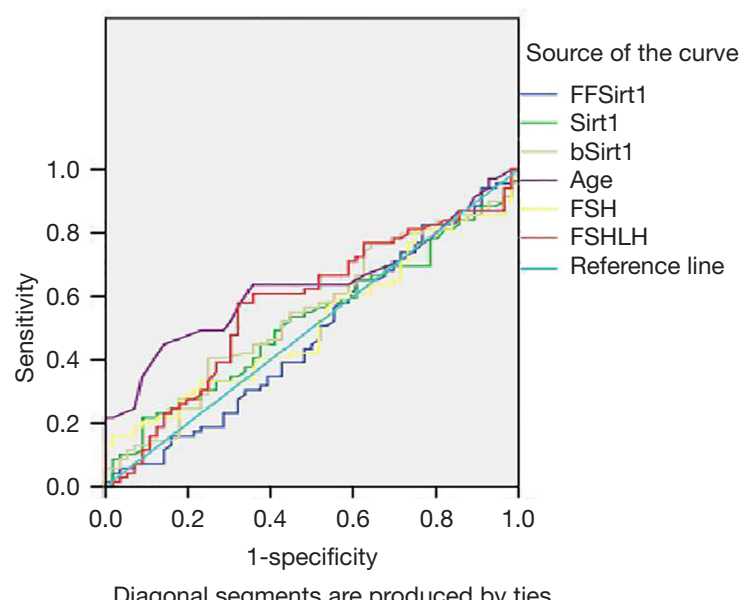

CR-AUC

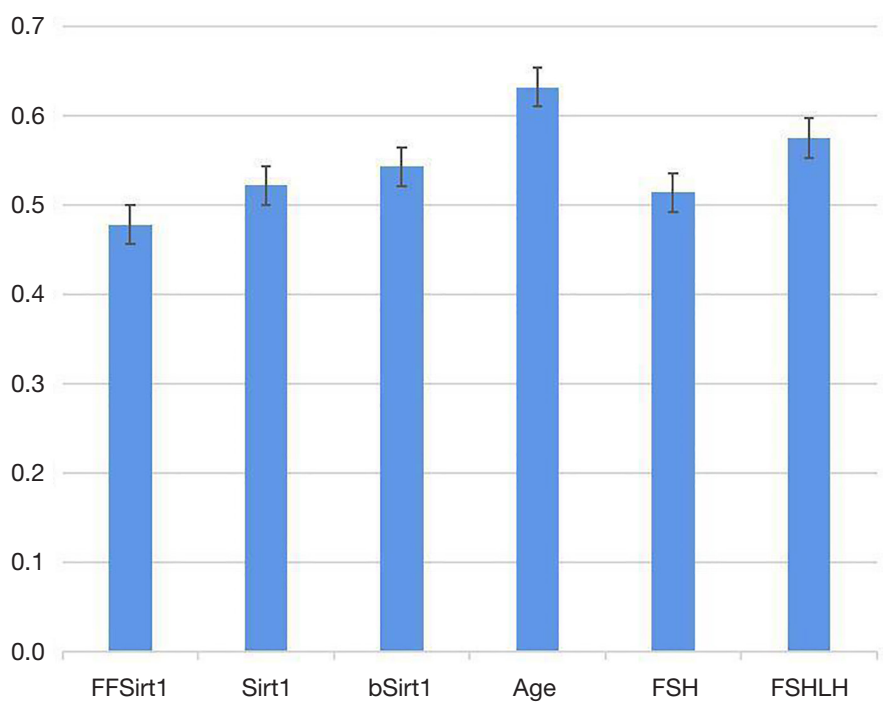

Figure 2 Age had statistical value in predicting cumulative clinical pregnancy, but FF SIRT1, serum SIRT1, FSH and FSH/LH had no statistical value in predicting cumulative clinical pregnancy. CR, cumulative pregnant rate; bSIRT1, base level of serum SIRT1; FFSIRT1, follicular fluid SIRT1; FSH, base level of follicle-stimulating hormone; LH, luteinizing hormone; SIRT, sirtuin; SIRT1, HCG injection day serum SIRT1; AUC, area under ROC curve; ROC, receiver operating characteristic curve. 
Table 2 Comparison of sensitivity and specificity of related sensitive parameters in predicting cumulative pregnancy outcomes of the patients

\begin{tabular}{|c|c|c|c|c|c|}
\hline Parameters & Area under ROC curve (AUC) & Sensitivity & Specificity & Critical point & $P$ value \\
\hline $\mathrm{AMH}$ & 0.686 & 0.36 & 0.94 & 3.31 & 0.000 \\
\hline AFC & 0.667 & 0.55 & 0.76 & 6.5 & 0.002 \\
\hline Age & 0.632 & 0.85 & 0.45 & 38.5 & 0.012 \\
\hline
\end{tabular}

bSIRT2, base level of serum SIRT2; AMH, anti-Müllerian hormone; AFC, antral follicular count; AUC , area under ROC curve ; ROC, receiver operating characteristic curve; SIRT, sirtuin.
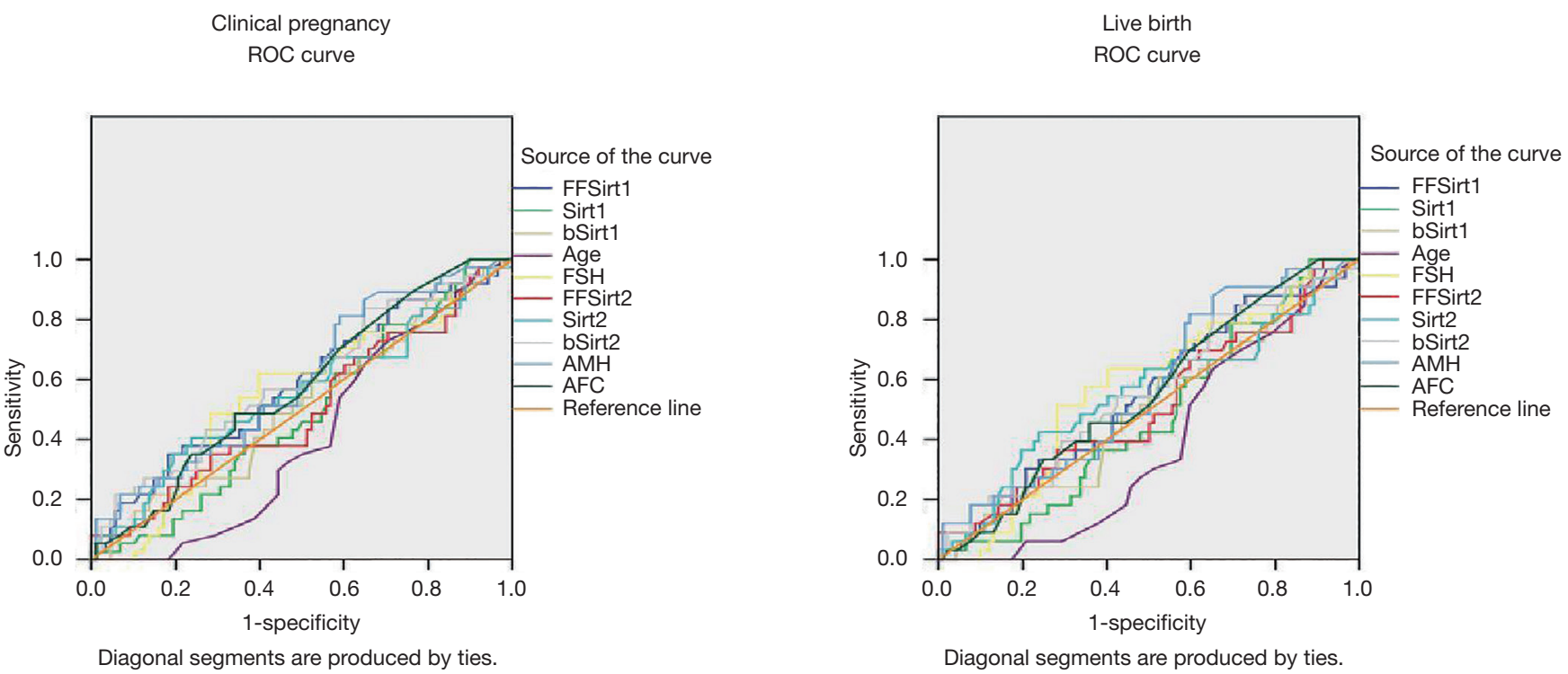

Figure 3 Serum and FF SIRT1, SIRT2, AFC, AMH, age, and basic FSH have no statistical value in predicting clinical pregnancy and live birth in assisted reproduction. AFC, antral follicular count; AMH, anti-Müllerian hormone; bSIRT1, base level of serum SIRT1; bSIRT2, base level of serum SIRT2; FFSIRT1, follicular fluid SIRT1; FFSIRT2, follicular fluid SIRT2; FSH, base level of follicle-stimulating hormone; SIRT, sirtuin; SIRT1, HCG injection day serum SIRT1; SIRT2, HCG injection day serum SIRT2; AUC, area under ROC curve; ROC, receiver operating characteristic curve.

during the mid-term spindle and cell division, ensuring normal cell division (38). A 2018 study (33) found that SIRT2 also participates in the regulation of normal oocyte meiosis devices by deacetylating BUBR1 and mediates the effects of advanced age on mouse oocyte quality. Xu (39) found that the expression of SIRT2 gradually decreased in a time-dependent manner during oocyte senescence; sirreal2 could significantly promote oocyte activation and increase the incidence of cytoplasmic fragmentation and spindle defects, while high levels of ROS, abnormal mitochondrial distribution, decreased ATP production, with $\Delta \psi \mathrm{m}$ loss being observed in oocytes exposed to SirReal2. Furthermore, overexpression of SIRT2 was found to induce
LC3-II accumulation and sequestosome-1 degradation. These findings suggest that SIRT2 inactivation is the key mechanism of cell aging, and SIRT2 inhibition contributes to the autophagy-dependent apoptosis of mature oocytes.

Previous studies have suggested that serum SIRT1 levels can predict neurodegenerative diseases such as Huntington's disease (HD), Parkinson's disease, and Alzheimer's disease (40). In different environments, SIRT2 may play a role in promoting or inhibiting tumor. Firstly, SIRT2 can inhibit the occurrence and development of many kinds of tumors, such as colon cancer, gastric cancer, lung cancer and breast cancer. In breast cancer cells, SIRT2 can inhibit the activity of antioxidant protein peroxiredoxin-1 (PRDX-1), 
Table 3 Logistic regression analysis of the correlation between infertility-related factors and cumulative clinical pregnancy

\begin{tabular}{|c|c|c|}
\hline Infertility-related factors & OR (95\% Cl) & $\mathrm{P}$ \\
\hline Age (years) & $0.972(0.890-1.062)$ & 0.528 \\
\hline BMI & $0.841(0.702-1.009)$ & 0.062 \\
\hline $\mathrm{FSH}$ & $0.991(0.834-1.178)$ & 0.920 \\
\hline $\mathrm{FSH} / \mathrm{LH}$ & $1.053(0.683-1.625)$ & 0.814 \\
\hline E2 & $0.991(0.970-1.013)$ & 0.417 \\
\hline $\mathrm{AMH}$ & $1.328(0.766-2.300)$ & 0.312 \\
\hline AFC & $0.953(0.805-1.128)$ & 0.576 \\
\hline Number of frozen embryos & $1.387(0.993-1.937)$ & 0.055 \\
\hline $\begin{array}{l}\text { Number of transferred } \\
\text { embryos }\end{array}$ & $1.478(1.053-1.272)$ & 0.165 \\
\hline FFSIRT1 & $1.052(0.851-2.568)$ & 0.228 \\
\hline FFSIRT2 & $0.974(0.894-1.060)$ & 0.539 \\
\hline HCG day SIRT1 & $1.040(0.968-1.117)$ & 0.282 \\
\hline HCG day SIRT2 & $1.048(0.949-1.157)$ & 0.358 \\
\hline bSIRT1 & $0.980(0.904-1.063)$ & 0.625 \\
\hline bSIRT2 & $1.101(1.000-1.212)$ & 0.049 \\
\hline
\end{tabular}

Statistically significant differences $(\mathrm{P}<0.05)$ are marked in italics type. $\mathrm{AMH}$, anti-Müllerian hormone; AFC, antral follicular count; $\mathrm{BMI}$, body mass index; FSH, follicle-stimulating hormone; bSIRT1, base level of serum SIRT1; bSIRT2, base level of serum SIRT2; E2, Estradiol; FFSIRT1, follicular fluid SIRT1; FFSIRT2, follicular fluid SIRT2; HCG day SIRT1, HCG injection day serum SIRT1; HCG day SIRT2, HCG injection day serum SIRT2; $\mathrm{LH}$, luteinizing hormone; SIRT, sirtuin. HCG, human chorionic gonadotropin; OR, odds ratio; $\mathrm{Cl}$, confidence interval.

enhance the sensitivity of breast cancer cells to ROSinduced DNA damage, and promote the apoptosis of breast cancer cells (41). SIRT2 can inhibit the expression of JMJD2A by binding to the promoter region of the JMJD2A gene, thereby inhibiting the proliferation, clone formation, and tumor growth of non-small cell cancer cells (42).

However, few studies have examined the relationship between serum and FF SIRT protein and reproduction. Bódis (36) extracted serum SIRT1 and SIRT6, and FF SIRT1, and FF SIRT6 on the 21st day before the ovulation promotion cycle and on the day of oocyte retrieval and evaluated their correlation with IVF/ICSI fertility outcome. The results showed that the serum SIRT1 level in the pregnancy group (22 patients) was significantly higher than that in the non-pregnancy group ( 8 patients), while the serum SIRT6 level was significantly lower in the pregnancy group both before and after ovulation promotion. After adjusting for confounding factors, the FF SIRT6 level was positively correlated with mature oocytes $(\mathrm{F}=6.609)$, while serum SIRT1 and SIRT6 levels were associated with clinical pregnancy ( $\mathrm{F}=10.008, \mathrm{~F}=5.268)$.

In this study, considering cumulative pregnancy can better reflect the clinical situation of patients after onetime egg retrieval, the study population was divided into a cumulative pregnancy group and a cumulative nonpregnancy group. The levels of bSIRT2, AMH, and AFC, along with the number of retrieved eggs, mature eggs, highquality embryos, and blastocyst formations in the pregnancy group were significantly higher than those in non-pregnancy group, but their age was lower than that in non-pregnancy group. The levels of serum and FF SIRT1, HCG day serum SIRT2, and FF SIRT2 showed no significant difference between the groups. Subsequent logistic regression analysis further indicated that bSIRT2 was associated with cumulative pregnancy. The ROC curve was used to further analyze the value of serum and FF SIRT1 and SIRT2 in predicting the outcome of assisted reproductive pregnancy, and only AMH, AFC, bSIRT2, and age showed statistical significance. The area under the curve of bSIRT2 was slightly higher than that of age, but slightly lower than that of $\mathrm{AMH}$ and $\mathrm{AFC}$, and the cutoff value was $29.95 \mathrm{IU} / \mathrm{L}$. Although the specificity was lower than that of $\mathrm{AMH}$ and $\mathrm{AFC}$, the sensitivity was higher. $\mathrm{AMH}$ and $\mathrm{AFC}$ are more likely to predict pregnancy outcome from the perspective of ovarian reserve and the number of follicles, while bSIRT2 may do so more from the perspective of egg quality and body state, because pregnancy is not only related to ovarian reserve, but also closely related to egg quality, uterine environment, and systemic status. According to the results, basal serum SIRT2 could be used to predict cumulative pregnancy outcome together with $\mathrm{AMH}, \mathrm{AFC}$, and age. It is convenient for clinicians to better evaluate the fertility of patients in clinical work. For patients with weak fertility, it is recommended to abandon treatment after multiple attempts of IVF/ICSI to avoid further costs, while for patients with better fertility assessment, it is recommended to actively accept assisted reproduction treatment. On the other hand, whether exogenous supplementation of SIRT2 agonists can improve the outcome of IVF/ICSI pregnancy is worth studying.

Our correlation analysis revealed a correlation between FF SIRT2, age, and AFC, and also indicated that the serum 


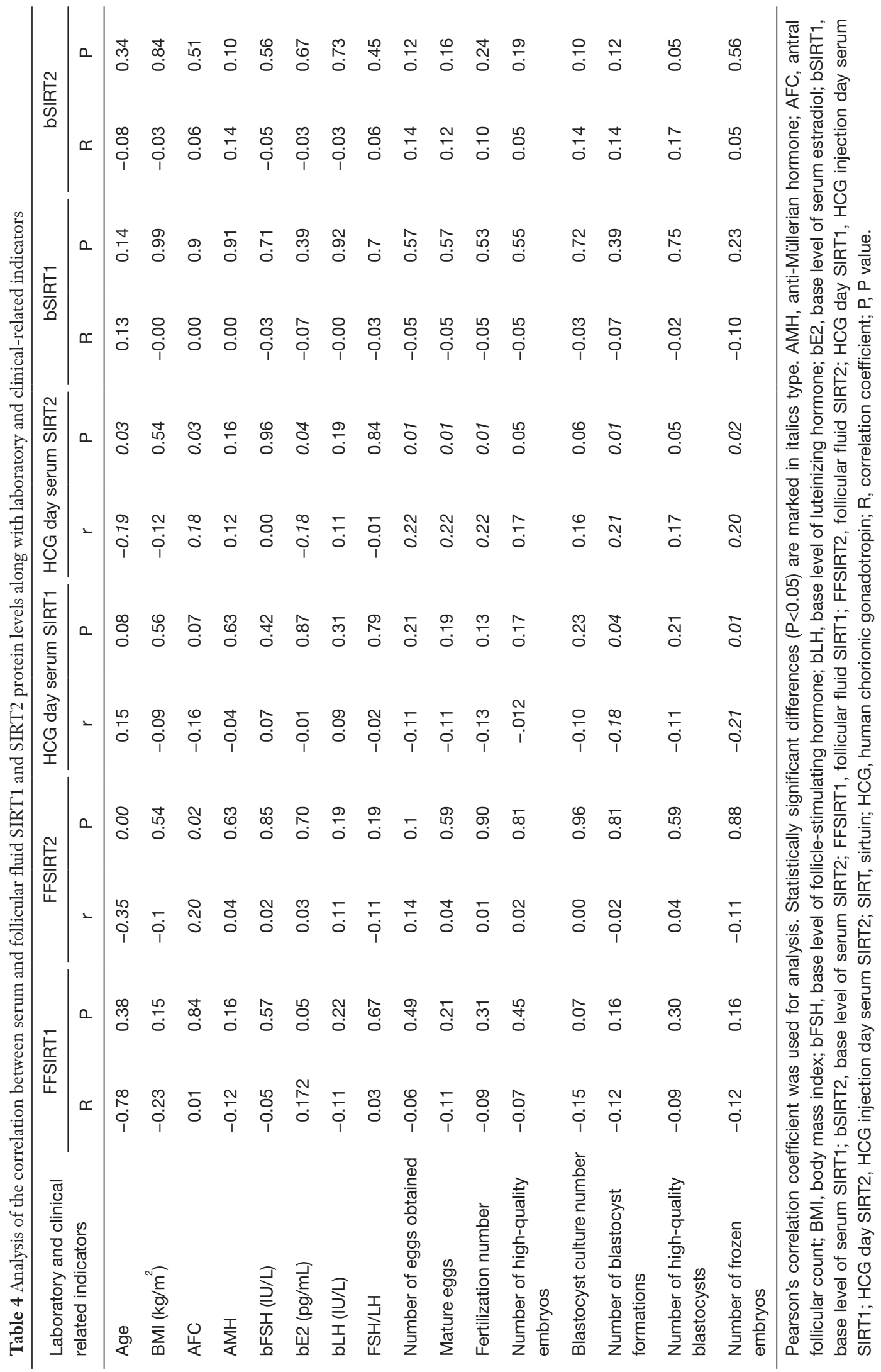


Table 5 Changes and correlation of SIRT1 and SIRT2 in serum and follicular fluid during assisted reproduction

\begin{tabular}{|c|c|c|c|c|c|c|}
\hline$n=125$ & \multicolumn{3}{|c|}{ SIRT1 } & \multicolumn{3}{|c|}{ SIRT2 } \\
\hline Mean \pm SD & $24.15 \pm 5.80^{a}$ & $33.84 \pm 7.03^{b}$ & $33.06 \pm 5.85^{b}$ & $22.45 \pm 5.68^{a}$ & $31.40 \pm 5.00^{b}$ & $31.32 \pm 4.93^{b}$ \\
\hline$P$ & & 0.000 & & & 0.00 & \\
\hline Correlation $(\mathrm{P})$ & & $>0.05$ & & & $>0.05$ & \\
\hline
\end{tabular}

FF, follicular fluid; HCG day, HCG injection day; HCG, human chorionic gonadotropin; Base, 2-5 days of menstruation; SIRT, sirtuin; SD, standard deviation. Different letters $(a, b)$ indicate that there is a significant difference between 2 values, while the same letter indicates no difference; $\mathrm{P}<0.05$ indicates that there is a significant difference.

level of SIRT1 (on HCG injection day) was negatively correlated with blastocyst formation. Furthermore, the serum levels of SIRT2 (HCG day) were positively correlated with AFC, the number of eggs, the number of matured eggs, the number of fertilizations, the number of blastocyst formations, and the number of frozen embryos, but were negatively correlated with age and basal E2 levels $(\mathrm{P}<0.05)$. SIRT1 has been demonstrated to both promote and inhibit cell proliferation (40). Xiong et al. (43) showed that the downregulation of SIRT1 expression inhibited apoptosis of mouse granulosa cells. This may also partially explain the results of HCG day serum SIRT1 in our study. Researchers including Bódis (36) have suggested that serum SIRT1 is negatively correlated with age on the day of egg collection, but not correlated with other indicators such as the number of oocytes retrieved, which differs from the results of our study. SIRT2 not only participates in oocyte meiosis, but also participates in a variety of inflammatory and oxidative pathways. SIRT2 interacts with NF- $\kappa \mathrm{B}$ in oxidative stress and regulates intracellular ROS levels which is also closely related to the quality of eggs and embryos $(40,44)$. These facts may explain why serum and FF SIRT2 influenced the results of our study, although more research is needed to verify this.

We also found that there was no significant difference between the bSIRT1, bSIRT2, serum SIRT1, and serum SIRT2 levels on the day of HCG injection, but the levels of SIRT1 and SIRT2 in FF were significantly lower than those in serum SIRT1 and SIRT2. There was no significant correlation between SIRT1 and SIRT2 in serum and FF. These results are consistent with those of Bódis (36). Serum SIRT1 and SIRT2 protein levels were not affected by ovulation induction drugs. The levels of SIRT1 and SIRT2 protein in FF may be secreted locally by the ovary independent from the levels of serum SIRT1 and SIRT2 protein.

\section{Limitations}

The main limitations of the study are its small sample size and the fact that only one ELISA method was used to detect the serum and FF concentration of SIRT1 and SIRT2. Longitudinal studies with larger sample sizes and more accurate detection methods are needed to clarify the correlation between serum and FF SIRT1 and SIRT2 and ovarian reserve, egg quality, embryo quality, and reproductive outcomes. Another limitation is that we did not detect the level of related indicators for ROS reactive and impaired mitochondrial function which are related to SIRT2. These issues will be addressed in future research.

\section{Conclusions}

This study found that the basal serum SIRT2, together with $\mathrm{AMH}, \mathrm{AFC}$, and age, could be used to predict cumulative pregnancy outcomes. However, serum and FF SIRT1, SIRT2, AMH, AFC, and age had no predictive value for clinical pregnancy and live birth of fresh embryo transfer. FF SIRT2 and serum SIRT2 on the day of HCG injection may be negatively correlated with age, but positively correlated with AFC. The serum SIRT1 level on HCG day was negatively correlated with the number of blastocysts and frozen embryos.

\section{Acknowledgments}

Funding: This work was supported by the Natural Foundation Project of Zhejiang Province (No. LQ15H030001).

\section{Footnote}

Reporting Checklist: The authors have completed the STARD reporting checklist. Available at http://dx.doi.org/10.21037/ atm-21-63 
Data Sharing Statement: Available at http://dx.doi. org/10.21037/atm-21-63

Conflicts of Interest: All authors have completed the ICMJE uniform disclosure form (available at http://dx.doi. org/10.21037/atm-21-63). The authors have no conflicts of interest to declare.

Ethical Statement: The authors are accountable for all aspects of the work in ensuring that questions related to the accuracy or integrity of any part of the work are appropriately investigated and resolved. All procedures performed in this study involving human participants were in accordance with the Declaration of Helsinki (as revised in 2013). This study was approved by the Research Ethics Committee of the First Affiliated Hospital, College of Medicine, Zhejiang University (No. 2017-734) and written consent was obtained from all the patients.

Open Access Statement: This is an Open Access article distributed in accordance with the Creative Commons Attribution-NonCommercial-NoDerivs 4.0 International License (CC BY-NC-ND 4.0), which permits the noncommercial replication and distribution of the article with the strict proviso that no changes or edits are made and the original work is properly cited (including links to both the formal publication through the relevant DOI and the license). See: https://creativecommons.org/licenses/by-nc-nd/4.0/.

\section{References}

1. Baker VL. Primary ovarian insufficiency in the adolescent. Curr Opin Obstet Gynecol 2013;25:375-81.

2. Izhar R, Husain S, Tahir S, et al. Occult Form of Premature Ovarian Insufficiency in Women with Infertility and Oligomenorrhea as Assessed by Poor Ovarian Response Criteria. J Reprod Infertil 2017;18:361-7.

3. Yao L, Zhang W, Li H, et al. The role of serum AMH and FF AMH in predicting pregnancy outcome in the fresh cycle of IVF/ICSI: a meta-analysis. Int J Clin Exp Med 2015;8:1755-67.

4. Frye RA. Phylogenetic classification of prokaryotic and eukaryotic Sir2-like proteins. Biochem Biophys Res Commun 2000;273:793-8.

5. Lim JH, Lee YM, Chun YS, et al. Sirtuin 1 modulates cellular responses to hypoxia by deacetylating hypoxiainducible factor 1alpha. Mol Cell 2010;38:864-78.

6. Liu Y, Dentin R, Chen D, et al. A fasting inducible switch modulates gluconeogenesis via activator/coactivator exchange. Nature 2008;456:269-73.

7. Molla MD, Dessie G, Akalu Y, et al. Hepatocellular Expression of SIRT1 and Its Effect on Hepatocellular Carcinoma Progression: A Future Therapeutic Perspective. Int J Hepatol 2020;2020:2374615.

8. Ramakrishnan G, Davaakhuu G, Kaplun L, et al. Sirt2 deacetylase is a novel AKT binding partner critical for AKT activation by insulin. J Biol Chem 2014;289:6054-66.

9. Wang T, Geng SL, Guan YM, et al. Deacetylation of metabolic enzymes by Sirt2 modulates pyruvate homeostasis to extend insect lifespan. Aging (Albany NY) 2018;10:1053-72.

10. Poulose N, Raju R. Sirtuin regulation in aging and injury. Biochim Biophys Acta 2015;1852:2442-55.

11. Sosnowska B, Mazidi M, Penson P, et al. The sirtuin family members SIRT1, SIRT3 and SIRT6: Their role in vascular biology and atherogenesis. Atherosclerosis 2017;265:275-82.

12. Khan M, Shah SA, Kim MO. 17beta-Estradiol via SIRT1/ Acetyl-p53/NF-kB Signaling Pathway Rescued Postnatal Rat Brain Against Acute Ethanol Intoxication. Mol Neurobiol 2018;55:3067-78.

13. Wang B, Zhang Y, Cao W, et al. SIRT2 Plays Significant Roles in Lipopolysaccharides-Induced Neuroinflammation and Brain Injury in Mice. Neurochem Res 2016;41:2490-500.

14. Chen X, Wales P, Quinti L, et al. The sirtuin-2 inhibitor AK7 is neuroprotective in models of Parkinson's disease but not amyotrophic lateral sclerosis and cerebral ischemia. PLoS One 2015;10:e0116919.

15. Jing E, Gesta S, Kahn CR. SIRT2 regulates adipocyte differentiation through FoxO1 acetylation/deacetylation. Cell Metab 2007;6:105-14.

16. Liu R, Dang W, Du Y, et al. SIRT2 is involved in the modulation of depressive behaviors. Sci Rep 2015;5:8415.

17. Zhang J, Wang C, Nie H, et al. SIRT2 plays a significant role in maintaining the survival and energy metabolism of PIEC endothelial cells. Int J Physiol Pathophysiol Pharmacol 2016;8:120-7.

18. McBurney MW, Yang X, Jardine K, et al. The mammalian SIR2alpha protein has a role in embryogenesis and gametogenesis. Mol Cell Biol 2003;23:38-54.

19. Tatone C, Di Emidio G, Barbonetti A, et al. Sirtuins in gamete biology and reproductive physiology: emerging roles and therapeutic potential in female and male infertility. Hum Reprod Update 2018;24:267-89. 
20. Liu M, Yin Y, Ye X, et al. Resveratrol protects against ageassociated infertility in mice. Hum Reprod 2013;28:707-17.

21. Furat Rencber S, Kurnaz Ozbek S, Eraldemir C, et al. Effect of resveratrol and metformin on ovarian reserve and ultrastructure in PCOS: an experimental study. J Ovarian Res 2018;11:55.

22. Yoo JY, Kim TH, Fazleabas AT, et al. KRAS Activation and over-expression of SIRT1/BCL6 Contributes to the Pathogenesis of Endometriosis and Progesterone Resistance. Sci Rep 2017;7:6765.

23. Ding YW, Zhao GJ, Li XL, et al. SIRT1 exerts protective effects against paraquat-induced injury in mouse type II alveolar epithelial cells by deacetylating NRF2 in vitro. Int J Mol Med 2016;37:1049-58.

24. Sablina AA, Budanov AV, Ilyinskaya GV, et al. The antioxidant function of the p53 tumor suppressor. Nat Med 2005;11:1306-13.

25. Hasegawa K, Wakino S, Yoshioka K, et al. Sirt1 protects against oxidative stress-induced renal tubular cell apoptosis by the bidirectional regulation of catalase expression. Biochem Biophys Res Commun 2008;372:51-6.

26. Grabowska W, Sikora E, Bielak-Zmijewska A. Sirtuins, a promising target in slowing down the ageing process. Biogerontology 2017;18:447-76.

27. Adhikari D, Liu K. Molecular mechanisms underlying the activation of mammalian primordial follicles. Endocr Rev 2009;30:438-64.

28. Adhikari D, Risal S, Liu K, et al. Pharmacological inhibition of mTORC1 prevents over-activation of the primordial follicle pool in response to elevated $\mathrm{PI} 3 \mathrm{~K}$ signaling. PLoS One 2013;8:e53810.

29. Chen Z, Kang X, Wang L, et al. Rictor/mTORC2 pathway in oocytes regulates folliculogenesis, and its inactivation causes premature ovarian failure. J Biol Chem 2015;290:6387-96.

30. Zhang J, Fang L, Lu Z, et al. Are sirtuins markers of ovarian aging? Gene 2016;575:680-6.

31. North BJ, Marshall BL, Borra MT, et al. The human Sir2 ortholog, SIRT2, is an NAD+-dependent tubulin deacetylase. Mol Cell 2003;11:437-44.

32. Zhang L, Hou X, Ma R, et al. Sirt2 functions in spindle organization and chromosome alignment in mouse oocyte meiosis. FASEB J 2014;28:1435-45.

33. Qiu D, Hou X, Han L, et al. Sirt2-BubR1 acetylation pathway mediates the effects of advanced maternal age on oocyte quality. Aging Cell 2018;17:e12698.

34. Michishita E, Park JY, Burneskis JM, et al. Evolutionarily conserved and nonconserved cellular localizations and functions of human SIRT proteins. Mol Biol Cell 2005;16:4623-35.

35. Kolthur-Seetharam U, Teerds K, de Rooij DG, et al. The histone deacetylase SIRT1 controls male fertility in mice through regulation of hypothalamic-pituitary gonadotropin signaling. Biol Reprod 2009;80:384-91.

36. Bódis J, Sulyok E, Koszegi T, et al. Serum and follicular fluid levels of sirtuin 1, sirtuin 6, and resveratrol in women undergoing in vitro fertilization: an observational, clinical study. J Int Med Res 2019;47:772-82.

37. Xiang Y, Xu J, Li L, et al. Calorie restriction increases primordial follicle reserve in mature female chemotherapytreated rats. Gene 2012;493:77-82.

38. North BJ, Verdin E. Interphase nucleo-cytoplasmic shuttling and localization of SIRT2 during mitosis. PLoS One 2007;2:e784.

39. Xu D, Jiang $\mathrm{X}, \mathrm{He} \mathrm{H}$, et al. SIRT2 functions in aging, autophagy, and apoptosis in post-maturation bovine oocytes. Life Sci 2019;232:116639.

40. Pais TF, Szego EM, Marques O, et al. The NADdependent deacetylase sirtuin 2 is a suppressor of microglial activation and brain inflammation. EMBO J 2013;32:2603-16.

41. Fiskus W, Coothankandaswamy V, Chen J, et al. SIRT2 Deacetylates and Inhibits the Peroxidase Activity of Peroxiredoxin-1 to Sensitize Breast Cancer Cells to Oxidant Stress-Inducing Agents. Cancer Res 2016;76:5467-78.

42. Xu W, Jiang K, Shen $M$, et al. SIRT2 suppresses non-small cell lung cancer growth by targeting JMJD2A. Biol Chem 2015;396:929-36.

43. Xiong F, Hu L, Zhang Y, et al. miR-22 inhibits mouse ovarian granulosa cell apoptosis by targeting SIRT1. Biol Open 2016;5:367-71.

44. Keefe DL, Marquard K, Liu L. The telomere theory of reproductive senescence in women. Curr Opin Obstet Gynecol 2006;18:280-5.

(English Language Editor: B. Draper)

Cite this article as: Yao LN, Zhang TF, Lin WQ, Jiang N, Cao HF, Li H, Qian JH. Value of serum and follicular fluid sirtuin (SIRT)1 and SIRT2 protein levels in predicting the outcome of assisted reproduction. Ann Transl Med 2021;9(4):343. doi: 10.21037/atm-21-63 